\title{
GT2020-1229
}

\section{DRAFT:REDUCED ORDER METHOD BASED ON AN ADAPTIVE FORMULATION AND ITS APPLICATION TO FAN BLADE SYSTEM WITH DOVETAIL JOINTS}

\author{
Jie. Yuan; Christoph. Schwingshackl, Loic. Salles \\ Vibration University Technology Centre \\ Department of Mechanical Engineering \\ Imperial College London \\ London, UK \\ Email: jie.yuan@imperial.ac.uk
}

\author{
Chian. Wong, Sophoclis. Patsias \\ Rolls Royce plc \\ Derby, UK
}

\section{ABSTRACT}

Localized nonlinearities due to the contact friction interfaces are widely present in the aero-engine structures. They can significantly reduce the vibration amplitudes and shift the resonance frequencies away from critical operating speeds, by exploiting the frictional energy dissipation at the contact interface. However, the modelling capability to predict the dynamics of such large-scale systems with these nonlinearities is often impeded by the high computational expense. Component mode synthesis (CMS) based reduced order modelling (ROM) are commonly used to overcome this problem in jointed structures. However, the computational efficiency of these classical ROMs are sometimes limited as their size is proportional to the DOFs of joint interfaces resulting in a full dense matrix. A new ROM based on an adaptive formulation is proposed in this paper to improve the CMS methods for reliable predictions of the dynamics in jointed structures. This new ROM approach is able to adaptively switch the sticking contact nodes off during the online computation leading to a significant size reduction comparing to the CMS based models. The large-scale high fidelity fan blade assembly is used as the case study. The forced response obtained from the novel ROM is compared to the state-of-the-art CMS based Craig-Bampton method. A parametric study is then carried out to assess the influence of the contact parameters on the dynamics of the fan assembly. The feasibility of using this proposed method for nonlinear modal analysis is also characterised.

\footnotetext{
*Address all correspondence to this author.
}

\section{INTRODUCTION}

Bladed disk in aero-engines have attracted tremendous attention from turbo-machinery industries and research communities for more than half a century. These rotating rotors are regarded as one of the fundamental factors that determine the overall performance of both civil and military engines [1]. Bladed disk systems are not just decisive on the aerodynamic efficiency of aero-engines, but also critical on structural efficiency, because these components contribute to about $30 \%$ of the overall weight of a gas turbine engine. Fan blade systems, as the largest bladed disk assembly in turbofan engines, provide over $60 \%$ thrust of modern jet engines [2,3]. The fan system consists of large metal or composite blades that are commonly attached to a disk via curved or straight dovetail roots. This design ensures easy assembly and safe load distribution, and also provides essential damping to the system. The fan system is widely regarded as one of the most critical bladed disk system of an aero-engine. It therefore has been carefully designed in aero-engine industries to achieve high aerodynamic and structural efficiency, and ensure a reliable high cycle fatigue (HCF) life [2]. Even with the use of robust design and advanced manufacturing techniques, these bladed disk may still experience some unexpected mechanical failures, mostly in the form of HCF, during their operational service [4]. Such a failure very likely occurs in the dovetail joint due to the fretting fatigue $[5,6]$. As a result, the contact interfaces are always protected by the dry lubricant film coating to improve the fatigue life of the fan blade system [7]. The solid lubricant Molydag 254 is mostly used, which consists of MoS2

Copyright (c) 2020 by Rolls Royce plc 
powder, carbon and other solid lubricants in a thermosetting phenolic resin [6]. The function of the coating is to effectively reduce the contact stress in the joint by lowering the contact friction coefficient in order to ensure the reliable service life. A number of research indicate that the friction coefficients can be reduced from about 0.8 to a value between 0.1 to 0.3 with the coated surface $[6,7]$.

Due to the important role the fan blade assembly plays in the turbo-engine, the dynamic stress in the fan blade system must be calculated accurately for a reliable estimation of service life. However, due to the complex nonlinear dynamic nature of the frictional joint as well as the coating effects at the fan blade root, the state-of-the-art linear vibration analysis often leads to an over-design of the components by ignoring the nonlinearities from the joint. It also results in an inaccurate prediction of the contact stress field for wear predictions. In order to further improve the fan blade root design, it would be therefore necessary to take these non-linearities into account at the preliminary design [8]. The use of finite element (FE) method to simulate such large dynamical systems is however often impeded by the large computational cost due to the intensive contact interfaces and strong inherit contact friction nonlinearities. Harmonic balance methods (HBM) provide a steady-state solution that can decrease the computational time by several orders to evaluate the most concerning steady state dynamic response [1]. Despite the improved efficiency with HBM, the computational expense involved in the simulations still remains as a challenge from an industrial perspective. The use of ROM techniques is widely recognized as an efficient and accurate way to integrate these contact frictional non-linearities into the high fidelity dynamic simulations $[9,10]$.

Sub-structuring techniques have been widely used to study global dynamics of large-scale structures. CMS technique are regarded as one of the main sub-structuring techniques that highlights that the substructures are reduced using their component modes [11]. They have been extensively used as reduced order modelling techniques for jointed structures with contact friction interfaces [11,12]. Rubin and Craig-Bampton (CB) method are two typical methods based on sub-structuring techniques [13,14]. A detailed review of these sub-structuring techniques can be found in [15]. As these methods retain the whole contact interface DOFs, it would be convenient to couple with the constitutive contact model to simulate high fidelity contact friction behaviour on the interface. The main drawback of these CMS methods is that the size of their reduced models largely depends on the size of contact interface that would compromise their computational efficiency [16]. Interface reduction techniques have been proposed to condense the numerous constraint modes mostly through a secondary modal reduction on interface DOFs $[17,18]$. As an extension, orthogonal polynomial series was then integrated with these interface reduction methods to greatly improve the computational efficiency of the evaluation of interface modes [16]. Proper orthogonal decomposition can also be considered as an independent ROM method [19]. It can generate a projection basis using a set of snapshots of a full non-linear system response. The adaptive ROM method was recently put forward to further increase the computational efficiency of simulating the dynamical behavior of the jointed structures [20,21]. The adaptive ROM method is essentially an improved CMS based ROM technique for the jointed structures with the microslip motion. The main characteristic of this method is that the size of the reduced system can be adaptively updated according to the number of the sliding contact nodes during the computation of forced frequency response. This automatic size update is achieved based on a new equation of motion that combines a linearised joint system with an internal variable that accounts the penalty terms from non-linear effects in the joint contact interface. Such a formulation allows the static mode associated to the sticking contact nodes to be removed adaptively, which would greatly reduce the size of reduced dynamical system. This adaptive ROM method can be also integrated with the efficient HBM framework to predict the most interesting steady-state frequency response. It has been demonstrated in [21] that the adaptive ROM method can perform much higher efficient simulations of jointed structures with micro-slip motion than classical CMS based methods. For the two simple cases considered in [21], the adaptive method can speed up the simulation by 50 times for the 2D case and 100 times for the 3D case. However, the use of this adaptive ROM method for a large scale assembly like fan bladed disk has not been investigated yet. The feasibility of using this method for nonlinear modal analysis is not known.

This paper will investigate the adaptive ROM method for the application to the large scale fan blade assembly with dovetail joints. A high fidelity of finite element fan bladed disk system is considered as a case study. The CMS based Craig-Bampton is employed to benchmark the results from the adaptive ROM method. The paper is organized as follows: The fan blade system and its linear dynamics is firstly presented; the formulation of the adaptive reduced order model and its implementation with HBM framework is then briefly described; it is followed by the comparison of forced frequency response and computational cost between the adaptive method and the Craig-Bampton method; the parametric study associated to the contact parameters is then described; after that, the characteristics of using the adaptive ROM method for the nonlinear modal analysis is presented followed by the conclusion.

\section{REFERENCE FAN BLADE SYSTEM Finite element model}

A full scale fan blade assembly for a turbofan jet engine is shown in Fig. 1a. The assembly is made up of a number of twisted blades in light blue and a disc in red. The blade and disc are connected through a curved dovetail joint. The whole assem- 


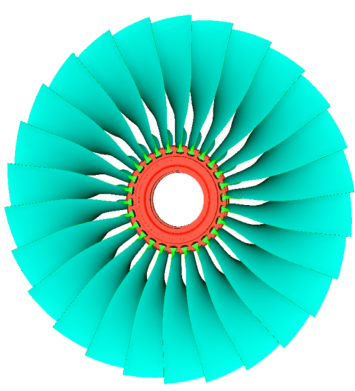

(a)

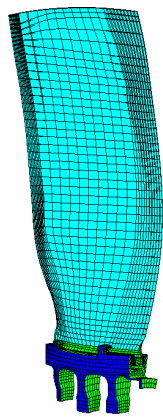

(b)

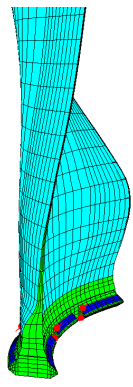

(c)
FIGURE 1: (a) Fan blade system assembly (b) Fan blade disc sector (c) The dovetail joint

bly is cyclic symmetric in nature. Figure $1 \mathrm{~b}$ shows one finite element sector of the fan bladed disk assembly in Fig. 1a. The blade has a low slenderness ratio, i.e 4, and an increasing twist from root to tip. Figure 1c shows the zoomed dovetail joint connecting the blade and disc. As discussed previously, this is one of crucially important mechanical parts in the fan blade system. The dovetail joint is used to integrate the blade and disc and effectively transfer the loading from the fan blade to the disc. With respect to the material, both the blade and disc are entirely made of titanium which is considered as a homogeneous and isotropic material. This high fidelity model was developed by the internal finite element software with quadratic hexahedral elements where each node has 3 DOFs. The matching mesh is used in the joint contact interface between the blade and disc. This node to node contact would enable us to apply the Jenkins contact element for accurate prediction of the contact friction force [22]. Each sector in the fan blade assembly contains 40,000 nodes, namely 120,000 DOFs. In terms of the DOFs in the dovetail joint, there are in total 208 contact pairs on the both side of the joint, which is in total 1248 contact friction DOFs. Figure 2. shows the distribution of the contact friction nodes in the $2 \mathrm{D}$ projected joint interface. It is worth noting that each contact pair has its own local coordinate system as the contact interface is in a highly curved shape.

Figure 3. displays the first four linearised vibrational modes of the sector of fan blade assembly. The joint interface between the blade and disc is linearised using the linear contact stiffness. The surfaces related to the cyclic symmetric boundaries are fully clamped by zeroing all the DOFs. It must be noted that the isolated sector may lose some features of a typical cyclic fan blade system. Although neglecting the cyclic symmetry will affect the stiffness of the disc, it will not significantly change the performance of the proposed reduction technique, which purely depends on the frictional behaviour on the interface. Applying

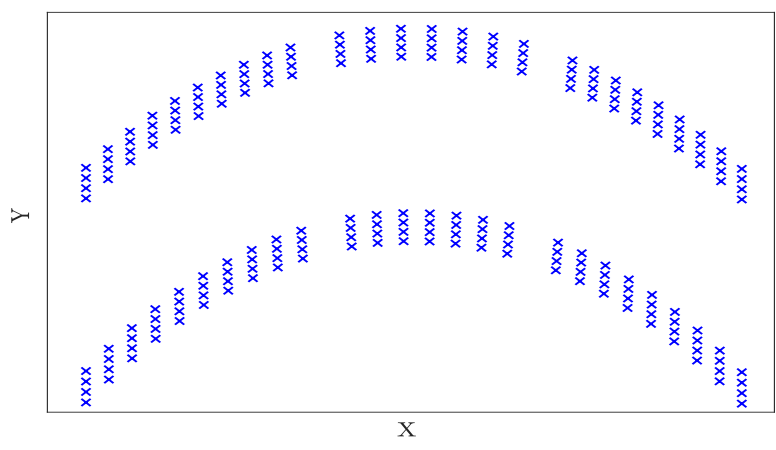

FIGURE 2: The distribution of the contact friction nodes in the 2D projected joint interface

the cyclic symmetry condition on a single sector would not further increase the number of nonlinear DOFs. The relative spacing of the first four frequencies is 1:1.3:2:4. As the fan blade is highly twisted due to the aerodynamic performance, the type of the first four mode shapes are mixed where there is no pure bending or torsion mode shape. It is however clear that out-ofplane flapping, edge-wise flapping and torsion motions dominate the first three modes respectively. In this paper, the effects of the dovetail joint on the dynamics of the first flapping mode is studied. It is also worth noting that the dynamics in a dovetail joint are strongly affected by both static and dynamic loads in operations. For a fan blade system, the simulations for static and dynamic analysis are separated to reduce the computational cost. The nonlinear dynamic analysis is performed based on the static equilibrium that is obtained from nonlinear static analysis using a commercial software. The preloading in contact elements therefore can be directly obtained. In this way, the effects of the static and dynamic loads on the contact conditions in the dovetail joints are both taken into account. The nonlinear static analysis is not presented in this paper.

\section{FORMULATION \\ Equation of motion}

The equation of motion for a structure with nonlinear contact friction interfaces can be written in the following form:

$$
\mathbf{M} \ddot{u}(t)+\mathbf{C} \dot{u}(t)+\mathbf{K} u(t)+\mathrm{F}_{n l}(u, t)=\mathrm{F}_{e}(t)
$$

where $u(t)$ is the generalized displacements vector; $\mathbf{M}, \mathbf{K}, \mathbf{C}$ are mass, stiffness and viscous damping matrices. $\mathrm{F}_{n l}(u, t)$ corresponds to the friction forces which is dependent on the displacement on the contact interfaces. $\mathrm{F}_{e}(t)$ is a vector of periodic external excitation forces. For the forced frequency response in this 


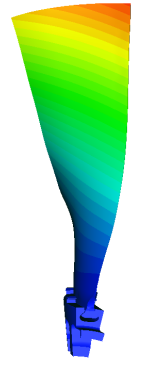

(a)

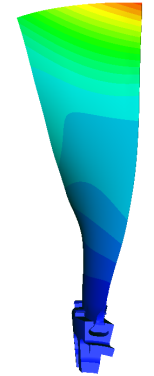

(b)

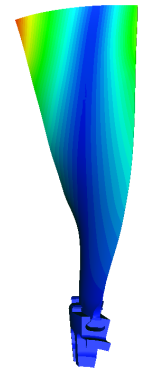

(c)

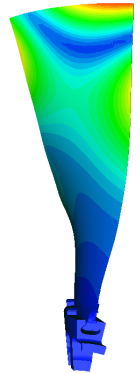

(d)
FIGURE 3: The first four mode shapes of the fan bladed disk

study, the force is applied at the tip of the fan blade normal to the sucking surface. For the nonlinear modal analysis, the excitation $\mathrm{F}_{e}(t)$ is set to zero making the system become autonomous.

A 3D node to node contact friction model is used to evaluate the contact friction force in the joint. Each friction element includes two decoupled 1D Jenkins elements to model a twodimensional in-plane tangential motion and a non-linear spring to apply contact laws in normal direction. Jenkins contact elements can be considered reasonably accurate to capture the realistic hysteretic loop of contact friction force. The contact parameters in Jenkins element are directly measurable from experiments [23]. Three contact friction states in a 3D node to node contact friction model can be briefly formulated as:

$$
\mathrm{F}_{T}(t)=\left\{\begin{array}{ccc}
0 & \text { if } F_{N}(t) \leq 0 \quad \text { (Separation) } \\
f_{T}(t) & \text { if } \begin{array}{l}
\mathrm{F}_{N}(t)>0 \\
\left|f_{T}(t)\right| \leq \mu \mathrm{F}_{N}(t)
\end{array} \text { (Stick) } \\
\mu \mathrm{F}_{N}(t) & \text { if } \begin{array}{l}
\mathrm{F}_{N}(t)>0 \\
\left|f_{t}(t)\right|>\mu \mathrm{F}_{N}(t)
\end{array} \text { (Slip) }
\end{array}\right.
$$

where $\mathrm{F}_{N}(t)$ and $\mathrm{F}_{T}(t)$ are the normal and tangential contact force; $\mu$ is the frictional coefficient; $f_{T}(t)$ is the tangential friction force when the nodes is in a contact condition. A detailed description of the 3D contact element with analytical expressions of the stiffness matrix can be found in [24]. The linear normal force $f_{N}^{L}(t)$ and tangential force $f_{T}^{L}(t)$ in a purely sticking condition at $i^{t h}$ contact node can be evaluated by:

$$
\begin{gathered}
f_{N}^{L}(t)=k_{n} \Delta u_{i}^{N}(t)+P_{i}^{N} \\
f_{T}^{L}(t)=k_{t} \Delta u_{i}^{T}(t)+P_{i}^{T}
\end{gathered}
$$

where $\Delta u_{i}^{N}(t)$ and $\Delta u_{i}^{T}(t)$ are the interface relative displacement in normal and tangential direction; $k_{t}, k_{n}$ are the tangential and normal contact stiffness for the contact surface; $P_{i}^{N}, P_{i}^{T}$ are the normal and tangential pre-loading on the $i^{\text {th }}$ contact node. The distribution of normal and tangential pre-loading can be obtained from non-linear static analysis in a commercial software assuming hard contact in contact surfaces.

\section{Modified equation of motion}

The adaptive ROM method is based on the linearised system where the whole contact interface is assumed to be in a sticking condition. The linearised system can be described as:

$$
\mathbf{M} \ddot{u}(t)+\mathbf{C} \dot{u}(t)+\mathbf{K}_{L}\left(k_{t}, k_{n}\right) u(t)=\mathrm{F}_{e}(u, t)
$$

where the $\mathbf{K}_{L}\left(k_{t}, k_{n}\right)$ is the linearised stiffness matrix of the jointed structure; $k_{t}, k_{n}$ are the tangential and normal contact stiffness for the contact surface. The prediction from the linearised system in Eq. (5). would not be accurate if any of the contact pairs is either in a slipping or separation condition. An internal variable $\Delta p$ is therefore introduced into the Eq. (5). to take into account the penalty from the non-linearities. The expression of the internal variable for $i^{\text {th }}$ contact pair in a local coordinate system can be formulated as:

$$
\Delta p^{i}=\left\{\begin{array}{cc}
\left(\mathrm{F}_{T}^{i}-k_{t} \Delta u_{i}^{T}\right) / k_{t} & \text { Tangential } \\
\left(\mathrm{F}_{N}^{i}-k_{n} \Delta u_{i}^{N}\right) / k_{n} & \text { Normal }
\end{array}\right.
$$

By adding the internal variable $\Delta p^{i}$ into the linearised system, the equation of motion for adaptive ROM can be written as:

$$
\left[\begin{array}{cc}
\mathbf{M} & \mathbf{0} \\
\mathbf{0} & \mathbf{0}
\end{array}\right]\left[\begin{array}{c}
\ddot{u} \\
\Delta \ddot{p}
\end{array}\right]+\left[\begin{array}{cc}
\mathbf{C} & \mathbf{0} \\
\mathbf{0} & \mathbf{0}
\end{array}\right]\left[\begin{array}{c}
\dot{u} \\
\Delta \dot{p}
\end{array}\right]+\left[\begin{array}{cc}
\mathbf{K}_{L} & \mathbf{B} \mathbf{K}_{\mathbf{J}} \\
\left(\mathbf{B K}_{\mathbf{J}}\right)^{T} & \mathbf{K}_{\mathbf{J}}
\end{array}\right]\left[\begin{array}{c}
u \\
\Delta p
\end{array}\right]=\left[\begin{array}{c}
\mathrm{F}_{e} \\
\mathrm{~F}_{n l}
\end{array}\right]
$$

where the $\mathbf{K}_{\mathbf{J}}$ is the linear joint stiffness matrix associated to the DOFs in contact interface; $\mathbf{B}$ is the signed Boolean matrix to transform the joint matrix into the global system matrix.

\section{Adaptive reduced order model}

The method for the first reduction of the system in Eq. (7). is based on the classical component mode synthesis technique. The transformation of the system from the physical to reduced space can be expressed as:

$$
\left[\begin{array}{c}
u \\
\Delta p
\end{array}\right]=\left[\begin{array}{ll}
\phi & \psi \\
\mathbf{0} & \mathbf{I}
\end{array}\right]\left[\begin{array}{c}
\eta \\
\Delta p
\end{array}\right]
$$




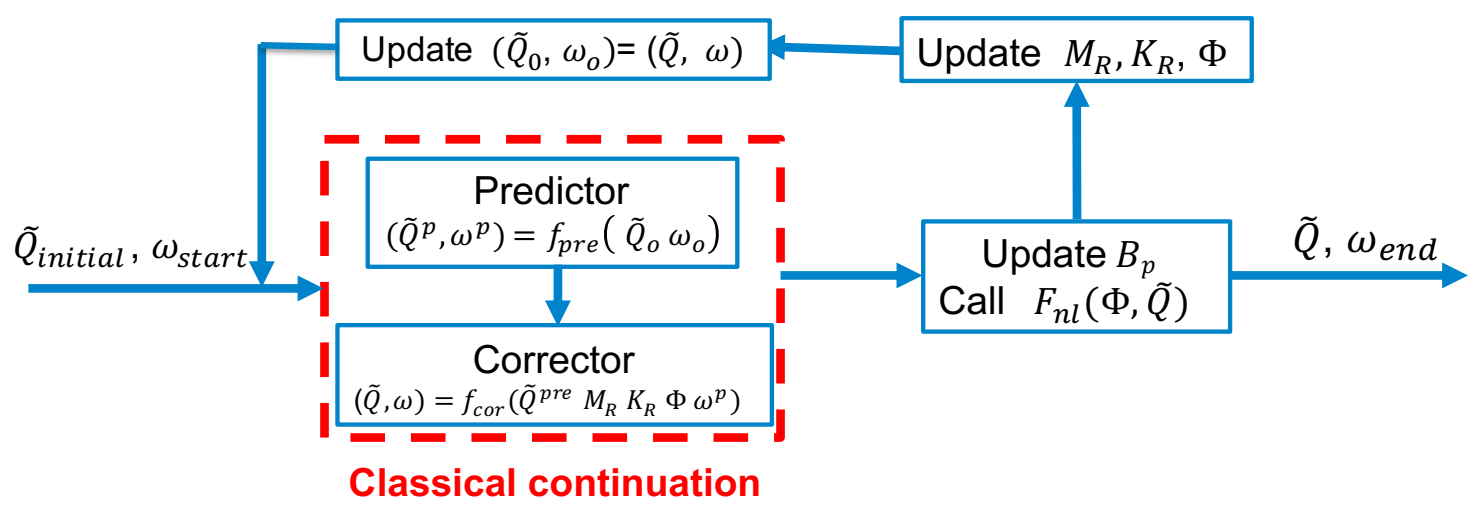

FIGURE 4: A flowchart of size updating procedure with harmonic balance method

where $\eta$ is the modal participation factors of the selected dynamic modes; $\phi$ are the vibrational modes of the linearised system; $\psi$ is the full set of static constraint modes associated to the DOFs in the contact friction interfaces. The details to obtain these reduced basis can be referred to $[20,21]$.

The adaptive reduced order model can then be constructed through the second reduction from Eq. (8). The second reduction consists in removing the static modes associated to purely sticking contact nodes from $\psi$. It is because these static modes are redundant in the reduced basis as the motion of the sticking nodes are included in the linearised dynamical modes. The second reduction can be easily achieved by removing the zero part from $\Delta p$ associated to the DOFs of the purely sticking nodes as defined in Eq. (6). The transformation matrix from the second reduction can be expressed as:

$$
\left[\begin{array}{c}
u \\
\Delta p
\end{array}\right]=\underbrace{\left[\begin{array}{ll}
\phi & \psi \\
\mathbf{0} & \mathbf{I}
\end{array}\right]\left[\begin{array}{cc}
\mathbf{I} & \mathbf{0} \\
\mathbf{0} & \mathbf{B}_{p}
\end{array}\right]}_{\Phi}\left[\begin{array}{c}
\eta \\
\Delta p_{R}
\end{array}\right],
$$

where $\Delta p_{R}$ is the non-zero part of $\Delta p ; \mathbf{B}_{p}$ is the Boolean matrix that help to abstract on the non-zero part of $\Delta p ; \Phi$ is the transformation matrix for the adaptive reduced order model. The size of $\Phi$ therefore depends on the contact condition of the contact nodes. It is worth to mention that such an adaptive ROM performs well in particular for a large number of nodes that remain in sticking condition, which is often the case for bladed disk connections. Using the modal projection with $\Phi$, the reduced mass and stiffness matrix can be written as:

$$
\mathbf{M}_{\mathbf{R}}=\Phi^{T} \mathbf{M}_{\mathbf{G}} \Phi, \mathbf{K}_{\mathbf{R}}=\Phi^{T} \mathbf{K}_{\mathbf{G}} \Phi
$$

\section{NONLINEAR VIBRATION SOLVER Harmonic balance method}

Multi-harmonic balance method is used to solve the reduced dynamic system. The main advantage of this method is to allow much faster computations of the nonlinear steady state response of the system compared to time-domain methods. The idea of HBM is to approximate the non-linear displacement $q(t)$ by a truncated Fourier series as:

$$
q(t)=\tilde{Q}_{0}+\sum_{i=1}^{n_{h}}\left(\tilde{Q}_{i}^{c} \cos m_{i} \omega t+\tilde{Q}_{i}^{s} \sin m_{i} \omega t\right)
$$

where $\tilde{Q}_{i}^{c, s}$ are cosine and sine harmonic coefficients for $i^{t h}$ harmonic; $\omega$ is the principal excitation frequency; $\tilde{Q}_{0}$ is the zero harmonic response; $n_{h}$ is the number of harmonics. Using such an approximation, the size of original unknown vector is then expanded by $2 n_{h}+1$ times. The framework of HBM mainly includes three components: iterative solver, Alternating Frequency Time (AFT) procedure and continuation technique. NewtonRaphson is used as the iterative solver. AFT technique is used to calculate the nonlinear contact friction in time domain. An inverse discrete Fourier transformation (IDFT) is applied on the displacements $\tilde{Q}(\omega)$ to get the displacements $\mathrm{Q}(t)$ in the time domain. From the contact equations, the contact forces $\mathrm{F}_{n l}(\mathrm{Q}, t)$ are determined in the time domain and a discrete Fourier transform (DFT) is then used to get the vector of Fourier coefficients of the nonlinear efforts $\tilde{\mathrm{F}}_{n l}(\omega)$. The procedure can be summarized as follows:

$$
\tilde{Q}(\omega) \stackrel{\text { IDFT }}{\longrightarrow} \mathrm{Q}(t) \stackrel{\text { Contact }}{\longrightarrow} \mathrm{F}_{n l}(\mathrm{Q}, t) \stackrel{\text { DFT }}{\longrightarrow} \tilde{\mathrm{F}}_{n l}(\omega)
$$

The continuation techniques is used to track the nonlinear dynamical response with tracking parameters e.g. frequency in 
forced frequency response and modal amplitude in nonlinear modal analysis. The details for these numerical techniques can be found in $[22,25,26]$.

\section{Automatic size updating}

The flowchart in Fig. 4. shows the process of the automatic size updating using the adaptive reduced order modelling formation. The computation starts with initial solution $\left(\tilde{Q}_{\text {initial }}, \omega_{\text {start }}\right)$ followed by the classical continuation calculation including stages of prediction and correction [25]. $\omega$ is the tracking parameter that is also included in the continuation process. A converged solution $\tilde{Q}$ is then obtained at a new frequency $\omega$. After that, the contact condition is re-evaluated to update the $\mathbf{B}_{p}$ for the next frequency point. This is achieved through the contact condition evaluation of the full contact nodes using the currently converged solution $(\tilde{Q}, \omega)$. The reduced system $\mathbf{M}_{\mathbf{R}}, \mathbf{K}_{\mathbf{R}}, \Phi$ is then updated with the identified $\mathbf{B}_{p}$ if the $\mathbf{B}_{p}$ is different from the one in the last frequency point. The old solution is then updated followed by the classical continuation process for evaluating a new frequency point. This iterative process continues until reaching the final frequency point $\omega_{\text {end }}$. More details can be found in the original paper in [21].

The updating algorithm is very efficient as the reduced system needs to be updated only once at most for a frequency point. The size of the reduced system remains constant during the iterative process in the correction stage. This is based on an assumption that it is reasonably accurate to predict the contact conditions for the next excitation frequency using the last converged solution. This assumption is valid as the region of the slipping or separation changes continuously and slowly in contact friction problems with the increase of excitation frequency or any other tracking parameters. The other big advantage of this algorithm is that the classical continuation part in original HBM framework keeps unchanged for using this new adaptive ROM approach. It is worth noting that the tracking parameter can also be a modal amplitude in the case of non-linear modal analysis.

\section{RESULTS AND DISCUSSIONS \\ Forced frequency response}

Fig.5. compares the forced frequency response between the adaptive ROM and CB method at the excitation level of $1 \mathrm{~N}$, $3 \mathrm{~N}, 5 \mathrm{~N}$ and $8 \mathrm{~N}$. One can observe that the resonance amplitude decreases slowly as the excitation level increases. This amplitude dependent behaviour is due to the increasing level of energy dissipation from the dovetail joint with the growing excitation level. The proposed adaptive method can exactly follow the trend of this amplitude dependent behaviour obtained from the CMS based CB method. However, the discrepancy in forced response between two methods can be found in the resonance amplitude at a high excitation level. The prediction of forced resonance re-

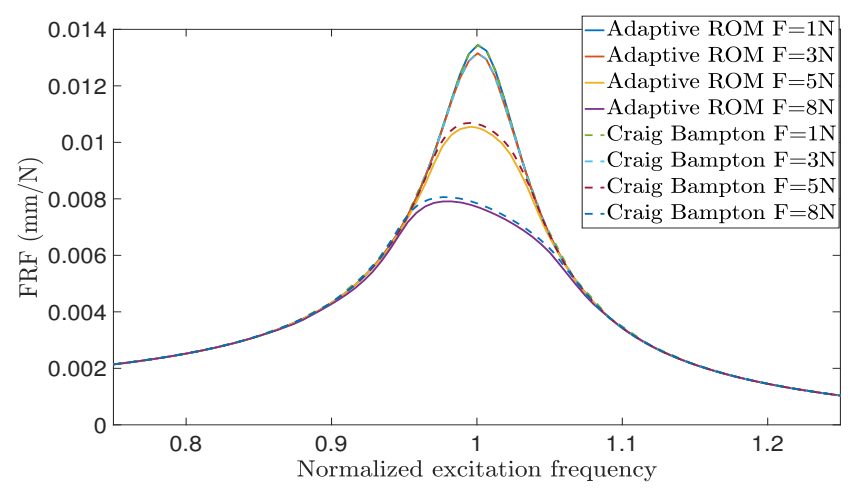

FIGURE 5: The comparison of FRFs between adaptive ROM and Craig-Bampton method

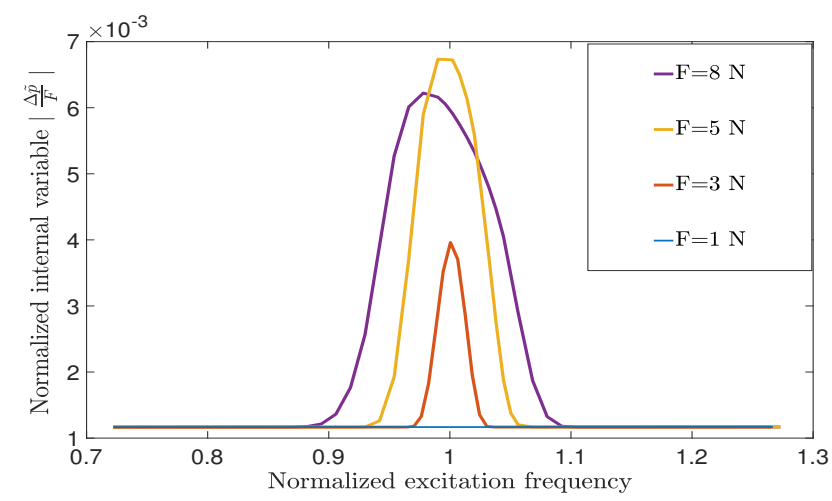

FIGURE 6: The value of normalized internal variable at different excitation levels

sponse from adaptive ROM is around $2.2 \%$ lower than that from $\mathrm{CB}$ method at the excitation level of $8 \mathrm{~N}$. It means the adaptive ROM overestimates the dissipation energy from contact surface that leads to lower vibrational level. This phenomena however has not been observed for small scale dynamical systems with 2D and 3D jointed beam structures studied in [21]. This may be due to the numerical errors associated to the automatic size updating algorithm because it predicts the contact condition at the next frequency point based on the current converged solution. Such inevitable numerical errors can make the updating algorithm mistaken some sticking nodes as partial slipping nodes. This fact suggests that an error estimator is necessary to be used in updating algorithm rather than purely depend on the zero terms in internal variable. Figure 6. compares the normalized internal variable over the same range of the excitation frequency at the corresponding excitation levels. The amount of internal variable indicates the level of non-linearities at different excitation levels. As expected, $\Delta \tilde{p}$ is zero at low excitation level $(1 \mathrm{~N})$ and at a frequency far away from the resonance as the system is in a linear 
region. With the increase of the excitation level, it becomes nonzero and increases significantly when the excitation frequency approaches to the resonance. It can clearly be seen that the width of the non-zero area is correlated to the non-linear zone shown in Fig.5.

\section{Parametric study}

Figure 7a. shows the change of the system size with the adaptive ROM model at the same four excitation levels. It can be found that the size of reduced system remain constant at the minimum size of 100 DOFs in the linear region. 100 is the total number of vibrational modes from the blade and disc. With the increase of the excitation level, the size of reduced system gradually increases for the excitation frequencies close to the resonance. The region of increased system size is corresponding to the nonlinear resonance area shown Fig.5. This indicates some of the contact nodes start to slip as the excitation force increases. One can also observe that the initial size of adaptive ROM is about 5000 in Fig.7a. This is because the DOFs of initial ROM is $1248 / 2+100=724$ DOFs, which is half number of nonlinear DOFs (1248) plus the number of vibrational modes (100). Since 3 harmonics is used in HBM (namely, 7 harmonic coefficients per DOF), the size of initial adaptive ROM therefore is $724 x 7=5086$. When it is in a full sticking condition, the size of the adaptive ROM is reduced to only 700. In terms of CMS based CB model, the total DOFs are kept constant at $1248+100=1348$ DOFs. Considering 7 harmonic coefficients per DOF, the total size for CMS ROM is kept constantly for any contact conditions at $1348 \times 7=9436$.

Fig. 7b. shows the detailed contact condition of the 208 contact nodes at different excitation levels. The red circle represents the contact node is in a stick-slip condition. Consistent with the size of reduced system in Fig.7a., as the excitation level increases, the number of stick-slip contact nodes increases significantly for the frequency points close to the resonance. The area of stick-slip nodes also expands toward to the further sides of the resonance frequency point.

Table 1. shows the computational speedup of the adaptive ROM against the CMS based CB method. 3 harmonics are constantly used for these two methods in both linear and nonlinear regions. This number was selected after a convergence study found that they were adequate to describe the beahviour at the interface. The adaptive ROM can significantly accelerate the computational speed for forced frequency response. It makes the simulation at least 19 times faster than the CB method at the high excitation level of $8 \mathrm{~N}$ when the contact nodes are mostly in a stick-slip condition. The speedup reaches the maximum of 87 when all the contact nodes are in a sticking condition at the excitation level of $1 \mathrm{~N}$. It is worth noting that the speedup factor is strongly influenced by the number of frequency steps where large parts of the contact remains in stick during forced frequency re- sponse analysis. If the whole interface is in slip, the problem would revert back to the unreduced ROM, eliminating any further speed up.

A parametric study is carried out to investigate the effect of varying contact friction properties and pre-loading on the nonlinear dynamics of the fan blade assembly. The computation for this parametric study is efficiently performed using the proposed adaptive ROM. Figure 8a. shows the influence of the varying friction coefficient on the FRFs at an excitation level of $5 \mathrm{~N}$. The variation of friction coefficients is due to the fretting wear on pre-loaded coated contact interface in dovetail joints. With the removal of dry film lubricant coating, the contact surface may eventually reach to full metal-to-metal contact that leads to dramatical increase in friction coefficients [6]. A wide range of the frictional coefficient between 0.1 and 0.9 is studied herein, which is similar to the range for the joint with dry film lubricant coating measured in [6]. We can see that the resonance of the forced response changes significantly with the increase of the friction coefficient. The resonance amplitude at $\mu=0.1$ is about 3 times lower than the amplitude at $\mu=0.8$. One can also observe the gradient of resonance peak at a low value of $\mu$ is much higher than that at a high value of $\mu$. This high sensitivity indicates it is crucially important to take into account the coating effects in the joint for the reliable prediction of the vibration levels. Figure $8 \mathrm{~b}$. shows the similar trend of the forced frequency response with the increase of the pre-loading level. It is obvious that, at the same excitation level, the sliding condition for a contact node becomes more difficult to reach.

Figure 10. shows the contact condition of all the joint interface nodes at different frictional coefficients. The red circle represents the stick-slip node; the blue cross represents the purely sticking node; the green circle represents the separating node. With the decrease of the frictional coefficient, the sliding starts from the middle of the outward contact surface and the edges of the inward surface. The number of stick-slip nodes then increase and expands continuously to the full contact interface. When $\mu=0.1$, some contact nodes appear separated in the middle of the outward contact surface.

\section{Damped nonlinear modal analysis}

The nonlinear modal analysis (NMA) is also carried out using the adaptive reduced order model. The concept of extended periodic motion is used to compute the resonance frequency and also damping properties directly with the increase of the modal amplitude $\alpha$. The formulation of the adaptive ROM and also the automatic size updating procedure is the essentially same as the forced frequency response except that external frequency force $\mathrm{F}_{e}$ is set to zero and the tracking parameter is the modal amplitude $\alpha$ instead of the frequency $\omega$. To solve this autonomous system with harmonic balance method, two additional normalization constrains are imposed as there would be two more unknown pa- 


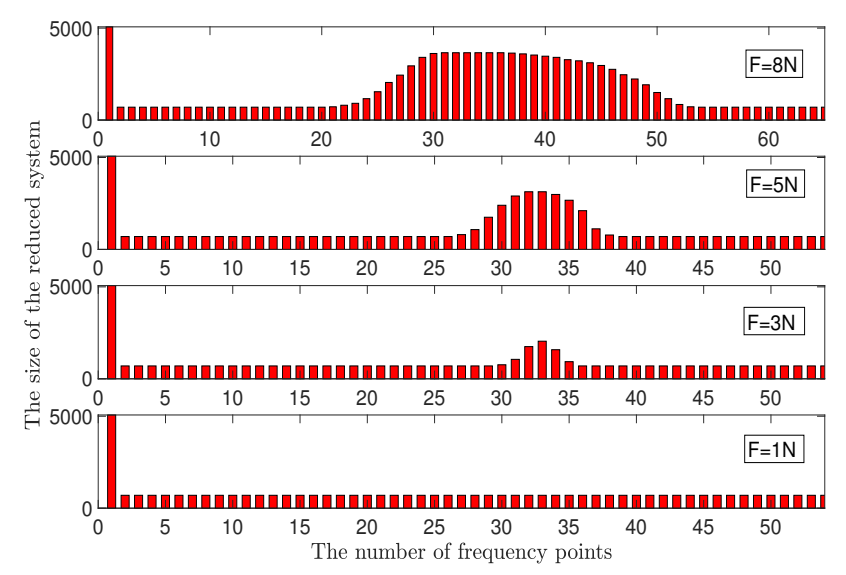

(a)

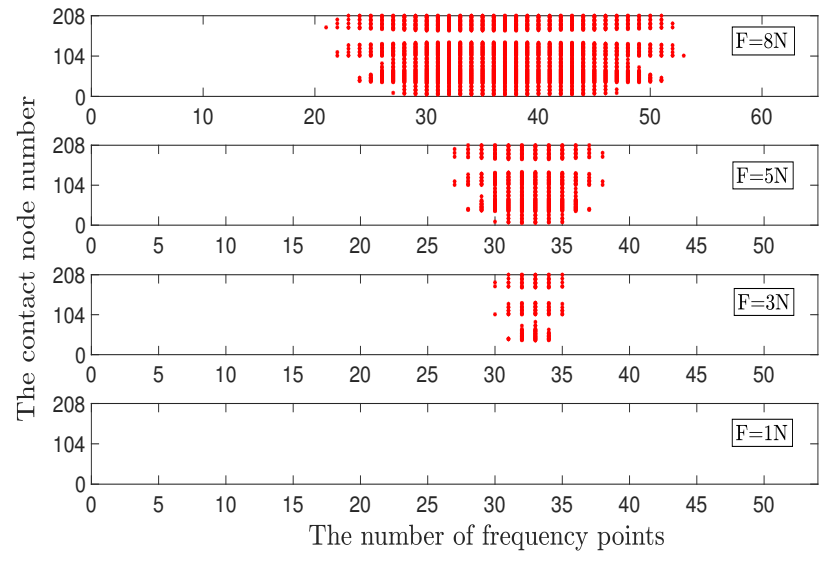

(b)

FIGURE 7: (a)The size of the adaptive reduced order model (b) The contact condition of interface nodes (Red cross: stick-slip node)

\begin{tabular}{|c|c|c|c|c|c|}
\hline & \multicolumn{2}{|c|}{ Adaptive ROM method } & \multicolumn{2}{c|}{ CMS based CB method } & Speedup \\
\hline Force Level & Time & Evaluations & Time & Evaluations & Time per Evaluation \\
\hline F=8N & $1239.7 \mathrm{~s}$ & 314 & $32180.9 \mathrm{~s}$ & 429 & $\mathbf{1 9}$ \\
\hline $\mathrm{F}=5 \mathrm{~N}$ & $356.16 \mathrm{~s}$ & 208 & $20737.8 \mathrm{~s}$ & 367 & $\mathbf{3 3}$ \\
\hline $\mathrm{F}=3 \mathrm{~N}$ & $145.69 \mathrm{~s}$ & 179 & $19796.3 \mathrm{~s}$ & 320 & $\mathbf{7 6}$ \\
\hline $\mathrm{F}=1 \mathrm{~N}$ & $121.27 \mathrm{~s}$ & 168 & 18588.9 & 297 & $\mathbf{8 7}$ \\
\hline
\end{tabular}

TABLE 1: Comparison of computational cost between the adaptive ROM method and CMS based CB method

rameters namely resonance frequency and damping. One is for mode normalization and the other is phase normalization. The methodology of the nonlinear normal mode analysis with extended periodic motion can be found in [26-28], which would not be detailed in the paper.

Figure 10a. shows the change of the resonance frequency and damping ratio with the modal amplitude. The modal amplitude corresponds to the level of energy involved in the autonomous system. The resonance frequency in red remains constant at a low modal amplitude up to 0.016 and then decreases proportionally with a further increase of modal amplitude $\alpha$. The damping ratio from energy dissipation behaves oppositely to the resonance frequency. It keeps constant at a low modal amplitude and then starts to increase significantly when the modal amplitude continues to increase from 0.016 . Figure 10b. shows the contact condition of the 208 contact nodes at different modal amplitude where the red cross indicates the contact node is in a stick-slip condition. As expected, the number of sliding contact nodes increases when the modal amplitude increases. Fig- ure 10c. compares the backbone curve (in black) obtained from the NMA with the resonance peaks from the forced frequency response analysis. It shows the damped nonlinear mode analysis is able to reasonably predict the resonance by crossing the resonance points in forced frequency analysis, especially at low excitation levels. However, there is a discrepancy when the excitation level goes up, which requires a further investigation in future. Figure 10d. shows the size of adaptive reduced order model for NMA. One can clearly find that the size of the system is proportional to the contact condition of joint interface nodes shown Fig.10b. Comparing to CMS based method, the size of reduced system can be significantly reduced at the low modal amplitudes when the joint is in a micro-slip motion.

\section{CONCLUSIONS}

This paper described a novel adaptive ROM technique to reduce the computational time for nonlinear vibration analysis of the fan bladed disk system with dovetail joints. This adaptive 


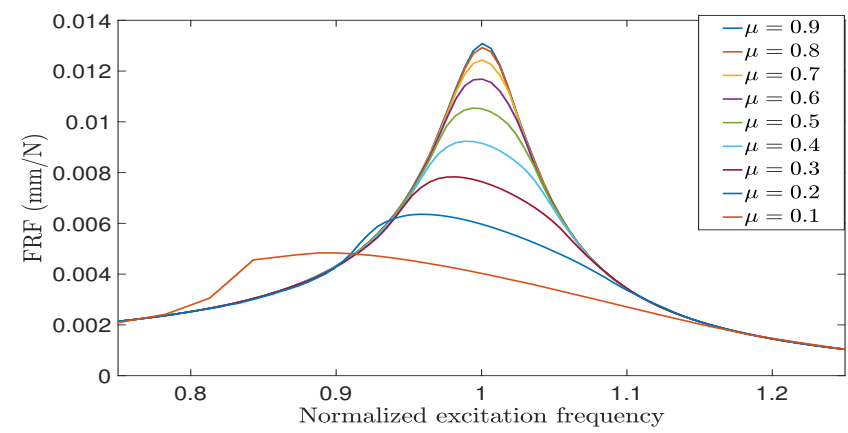

(a)

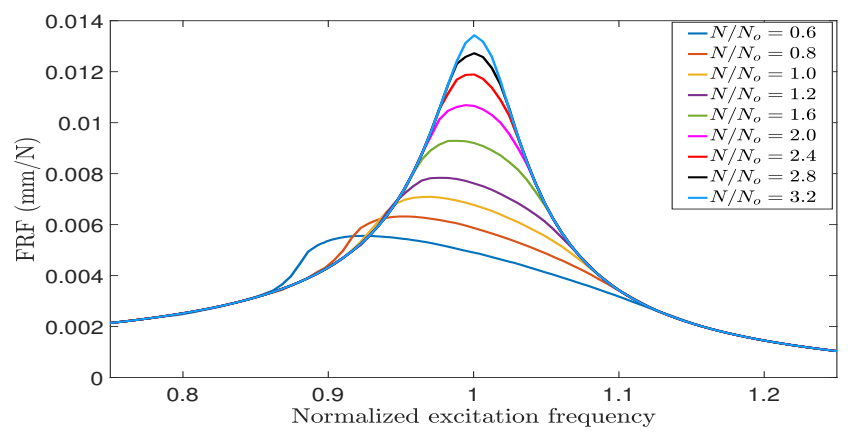

(b)

FIGURE 8: The forced frequency response with the varying (a) friction coefficient (b) static normal loading

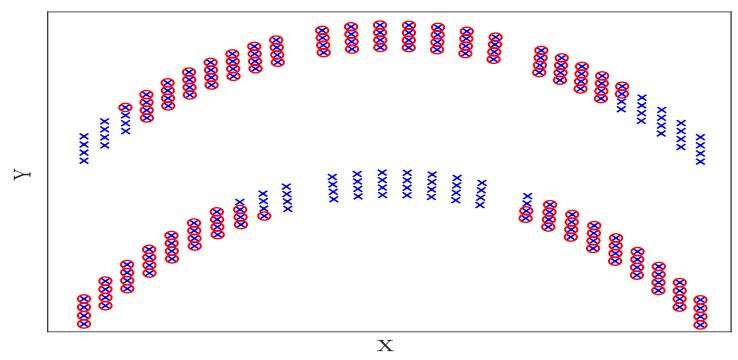

(a)

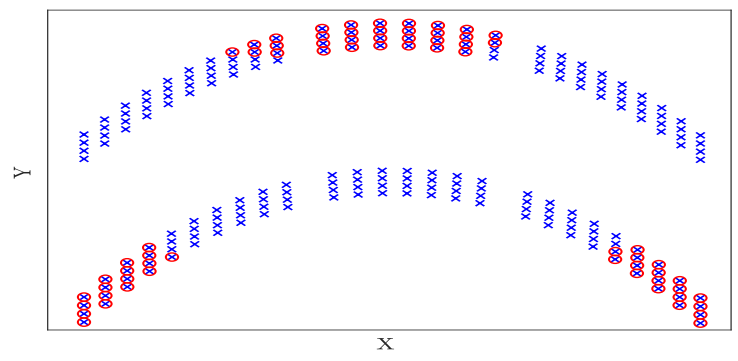

(c)

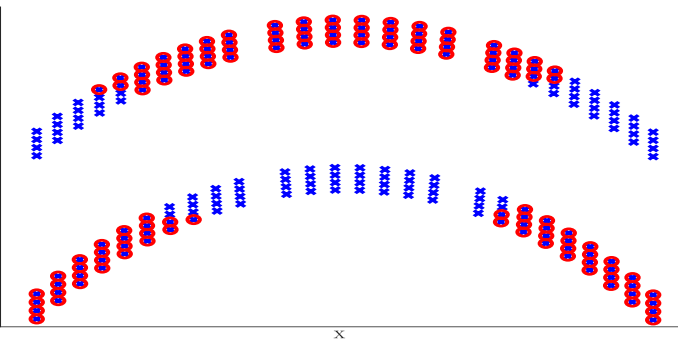

(b)

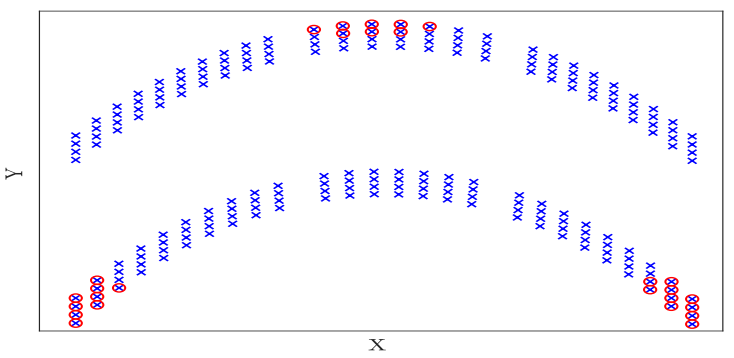

(d)

FIGURE 9: The resonance contact condition of contact joint node in the 2D projected joint surface ( Stick-slip condition in Red; Full stick condition in Blue; Separation in Green) (a) $\mu=0.1$ (b) $\mu=0.3$ (c) $\mu=0.5$ (d) $\mu=0.7$

ROM method is essentially an improved CMS technique for the jointed structure with micro-slip motion. It is based on an adaptive equation of motion that combines a linearised joint system with an internal variable that accounts the penalty terms from nonlinear effects in joint interface. The case study has shown that this novel ROM technique is able to adaptively update the size of reduced system according to the contact condition of interface nodes in forced frequency response analysis and also damped nonlinear modal analysis. Comparing to the CMS based CB method, the proposed method can make the simulations 19 to 87 times faster at different excitation levels. The high computational speedup is achieved when the joint is in a micro-slip motion as most contact nodes are in sticking conditions, and influenced by the number of frequency steps away from the resonance. It is because the system can be further reduced significantly by removing the static modes associated to these sticking 


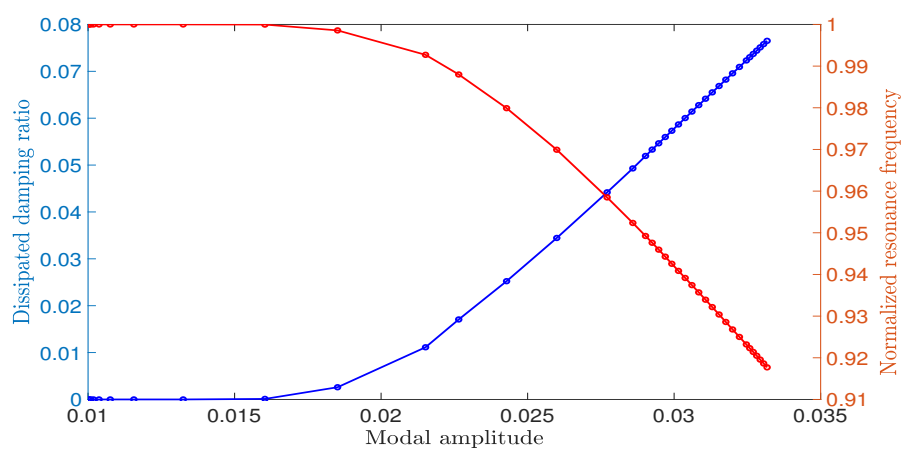

(a)

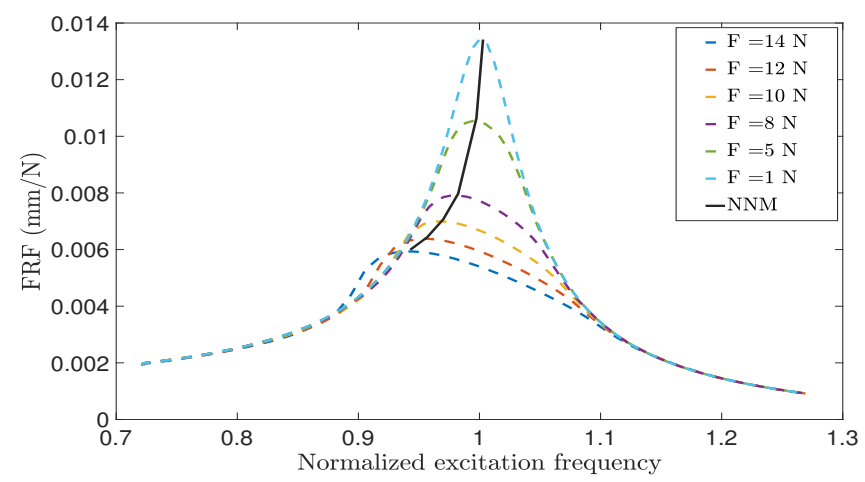

(c)

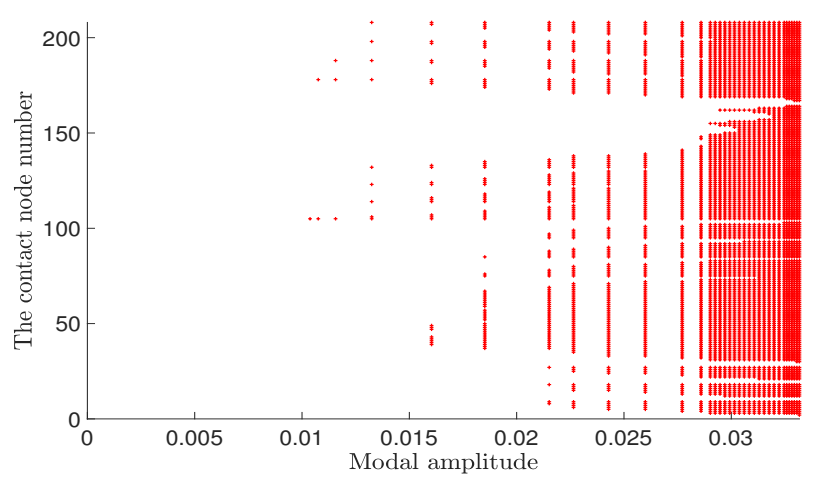

(b)

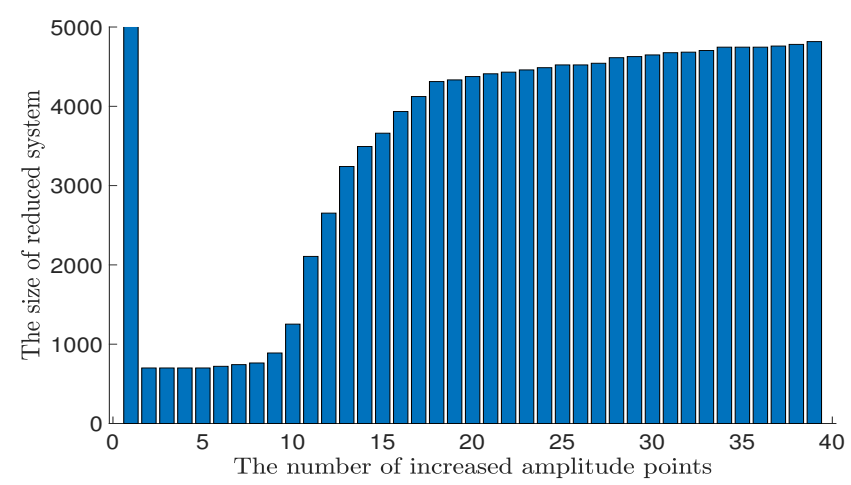

(d)

FIGURE 10: (a) Resonance frequency and dissipated damping ratio (b) The stick-slip condition of joint contact nodes (c) Comparison of between the backbone curve from NMA and FRFs (d) The size of the adaptive ROM

nodes in the transformation matrix. The proposed method also accurately captures the forced response over a wide range of excitation levels. For the case of strong friction damping, the size of adaptive ROM converges to the size of classical CMS ROM as most contact nodes are in a slipping condition. The parametric study showed that the coating in dovetail joints that leads to varying friction coefficients, as well as the change in the contact preloading, have a great influence on the dynamics of the fan blade system. The dynamic response can be three times lower with the dry film lubricant coating in terms of the resonance response. The paper also demonstrated that the adaptive ROM technique can be used for the NMA to directly compute the damping and resonance frequency. The size of this reduced autonomous system is automatically updated with the increase of modal amplitude leading to a huge size reduction at a low modal amplitude. The backbone curve obtained from NMA can accurately capture the resonance peaks in forced frequency response.

\section{ACKNOWLEDGMENT}

The authors are grateful to the support of Rolls-Royce plc and Innovate UK through GEMiniDS WP3 project 113088 and EPSRC through SYSDYMATS WP3 project R032793. L. Salles thanks Rolls-Royce plc and the EPSRC for the support under the Prosperity Partnership Grant "Cornerstone: Mechanical Engineering Science to Enable Aero Propulsion Futures" EP/R004951/1.

\section{REFERENCES}

[1] Krack, M., Salles, L., and Thouverez, F., 2017. "Vibration prediction of bladed disks coupled by friction joints". Archives of Computational Methods in Engineering, 24(3), Jul, pp. 589-636.

[2] Amoo, L. M., 2013. "On the design and structural analysis of jet engine fan blade structures". Progress in Aerospace Sciences, 60, pp. 1-11.

[3] Yuan, J., Scarpa, F., Allegri, G., Titurus, B., Patsias, S., and 
Rajasekaran, R., 2017. "Efficient computational techniques for mistuning analysis of bladed discs: A review". Mechanical Systems and Signal Processing, 87, pp. 71-90.

[4] Ewins, D., 2010. "Control of vibration and resonance in aero engines and rotating machinery-an overview". International Journal of Pressure Vessels and Piping, 87(9), pp. 504-510.

[5] Rajasekaran, R., and Nowell, D., 2006. "Fretting fatigue in dovetail blade roots: Experiment and analysis". Tribology international, 39(10), pp. 1277-1285.

[6] Fouvry, S., and Paulin, C., 2014. "An effective friction energy density approach to predict solid lubricant friction endurance: Application to fretting wear". Wear, 319(1-2), pp. 211-226.

[7] Barman, K., Shipway, P., Voisey, K., and Pattinson, G., 2018. "The role of a thermally sprayed cuniin underlayer in the durability of a dry-film lubricant system in fretting-a phenomenological model". Tribology International, 123, pp. 307-315.

[8] Seinturier, E., 2007. "Forced response computation for bladed disks industrial practices and advanced methods". Lecture Series-Von Karman Institute For Fluid Dynamics, 2, p. 5.

[9] Brake, M. R. W., Groß, J., Lacayo, R. M., Salles, L., Schwingshackl, C. W., Reuß, P., and Armand, J., 2018. Reduced Order Modeling of Nonlinear Structures with Frictional Interfaces. Springer International Publishing, Cham, pp. 427-450.

[10] Petrov, E., 2011. "A high-accuracy model reduction for analysis of nonlinear vibrations in structures with contact interfaces". Journal of Engineering for Gas Turbines and Power, 133(10), p. 102503.

[11] Battiato, G., Firrone, C., Berruti, T., and Epureanu, B., 2018. "Reduction and coupling of substructures via gramschmidt interface modes". Computer Methods in Applied Mechanics and Engineering, 336, pp. 187-212.

[12] Zucca, S., and Epureanu, B. I., 2018. "Reduced order models for nonlinear dynamic analysis of structures with intermittent contacts". Journal of Vibration and Control, 24(12), pp. 2591-2604.

[13] Rubin, S., 1975. "Improved component-mode representation for structural dynamic analysis". AIAA journal, 13(8), pp. 995-1006.

[14] Craig, R., and Bampton, M., 1968. "Coupling of substructures for dynamic analyses". AIAA journal, 6(7), pp. 13131319.

[15] Yuan, J., El-Haddad, F., Salles, L., and Wong, C., 2019. "Numerical assessment of reduced order modeling techniques for dynamic analysis of jointed structures with contact nonlinearities". Journal of Engineering for Gas Turbines and Power, 141(3), p. 031027.

[16] Carassale, L., and Maurici, M., 2018. "Interface reduction in craig-bampton component mode synthesis by orthogonal polynomial series". Journal of Engineering for Gas Turbines and Power, 140(5), p. 052504.

[17] Castanier, M. P., Tan, Y.-C., and Pierre, C., 2001. "Characteristic constraint modes for component mode synthesis". AIAA journal, 39(6), pp. 1182-1187.

[18] Tran, D.-M., 2001. "Component mode synthesis methods using interface modes. application to structures with cyclic symmetry". Computers \& Structures, 79(2), pp. 209-222.

[19] Jain, S., 2015. "Model order reduction for non-linear structural dynamics".

[20] Yuan, J., Salles, L., Wong, C., and Patsias, S. "A novel penalty-based reduced order modelling method for dynamic analysis of joint structures". In IUTAM Symposium on Model Order Reduction of Coupled Systems, Stuttgart, Germany, May 22-25, 2018.

[21] Yuan, J., Salles, L., El Haddad, F., and Wong, C., 2020. "An adaptive component mode synthesis method for dynamic analysis of jointed structure with contact friction interfaces". Computers \& Structures, 229, p. 106177.

[22] Pesaresi, L., Salles, L., Jones, A., Green, J., and Schwingshackl, C., 2017. "Modelling the nonlinear behaviour of an underplatform damper test rig for turbine applications". Mechanical Systems and Signal Processing, 85, pp. $662-$ 679.

[23] Fantetti, A., Tamatam, L., Volvert, M., Lawal, I., Liu, L., Salles, L., Brake, M., Schwingshackl, C., and Nowell, D., 2019. "The impact of fretting wear on structural dynamics: Experiment and simulation". Tribology International, 138, pp. 111-124.

[24] Salles, L., Blanc, L., Thouverez, F., Gouskov, A. M., and Jean, P., 2009. "Dynamic analysis of a bladed disk with friction and fretting-wear in blade attachments". In ASME Turbo Expo 2009: Power for Land, Sea, and Air, American Society of Mechanical Engineers, pp. 465-476.

[25] Sarrouy, E., and Sinou, J.-J., 2011. "Non-linear periodic and quasi-periodic vibrations in mechanical systems-on the use of the harmonic balance methods". In Advances in Vibration Analysis Research. InTech.

[26] Sun, Y., Yuan, J., Pesaresi, L., and Salles, L., 2018. "Nonlinear vibrational analysis for integrally bladed disk using frictional ring damper". In Journal of Physics: Conference Series, Vol. 1106, IOP Publishing, p. 012026.

[27] Krack, M., 2015. "Nonlinear modal analysis of nonconservative systems: Extension of the periodic motion concept". Computers and Structures, 154, pp. 59-71.

[28] Sun, Y., Yuan, J., Pesaresi, L., Denimal, E., and Salles, L., 2020. "Parametric study and uncertainty quantification of the nonlinear modal properties of frictional dampers". Journal of Vibration and Acoustics. 